\title{
A novel design of an air-cushion vehicle and its implementation
}

\author{
Zsolt Molnár ${ }^{1 *}$, Timotei István Erdei ${ }^{2}, N w a c h u k w u$ C. Obinna ${ }^{3}$, Géza $\mathrm{Husi}^{4}$ \\ ${ }^{1}$ University of Debrecen, zsolt.molnar94@gmail.com \\ ${ }^{2}$ University of Debrecen, timoteierdei@gmail.com \\ ${ }^{3}$ University of Debrecen, nwachukwucobinna@gmail.com \\ ${ }^{4}$ University of Debrecen, husigeza@eng.unideb.hu
}

\begin{abstract}
Recent advancements of unmanned aerial vehicles (UAVs) has led to an increase in public acceptance and availability of manufacturing technologies for these vehicles. This paper is concerned with the development of an air-cushion vehicle (ACV) utilizing the aforementioned manufacturing technologies. Exploiting similarities between UAVs and ACVs, and compensating for their differences, a unique hovercraft is designed and fabricated. The goal of the project is designing an ACV which can be controlled as an UAV without the dangers of easily damaging it. An overview of the development procedure of the hardware, software and implementation of the design is presented within this text.
\end{abstract}

\section{Introduction}

Currently, there is a vast amount of commercially available UAVs (Unmanned Aerial Vehicles). However these platforms tend to be closed source, and are of relatively high cost. In addition, making modifications to these UAVs is limited. In the midst of these limitations, it is still possible to design a drone which consists of an open-source controller. The open-source controllers utilized within the framework of this paper, include an Arduino and a Linux operating system-based controller.

The University of Debrecen, Faculty of Engineering, Department of Electrical Engineering and Mechatronics, Building Mechatronic Research Centre, G. Husi [1] made provision for all the necessary equipment required by this research project.

\section{Design considerations}

Several types of quadrotors has been developed within the research centre. By analysing the construction of these quadrotors, and on the basis of ACV (Air-Cushion Vehicle) design requirements, a layout of the hovercraft presented in this paper is achieved.

In comparison to classical hovercraft, S.A.M. Ali [2], flight is not necessary to achieve translational inertial motion of the presently proposed hovercraft. Furthermore, the proposed hovercraft features less navigation power consumption and is easily repaired. For these reasons, the lifecycle of this hovercraft is estimated at three times the average lifecycle of a quadrotor.

The first step of design was to find low density materials, and produce a Google Sketch Up 3D model of the hovercraft's geometry.

Google Sketch Up [3] software was very useful in enabling modifications of the $3 \mathrm{D}$ model to be made quickly and precisely. It is interesting to note that with the hovercraft's 3D model, one may develop a Computer Numerical Control (CNC) prototype to cut out the materials.

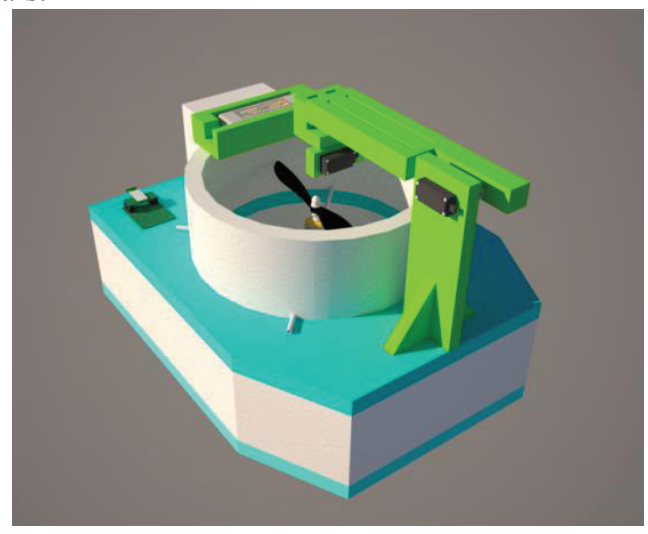

Fig. 1. 3D model of hovercraft

\footnotetext{
* Corresponding author: zsolt.molnar94@gmail.com
} 


\section{Construction materials}

The hovercraft houses three brushless DC (Direct Current) motors, and two of which provide the vehicle's buoyancy. They rotate in opposite directions, so that net torque generated by the motors is equals to zero. As a result, the vehicle is able to achieve vertical thrust, without redundant inertial rotation. Horizontal thrust is induced by the third brushless DC motor, which is housed at the rear of the vehicle. In order to optimize lift control, the motors responsible for lift generation are placed at the vehicle's centre.

On board the hovercraft is a controllable robot arm which contains within its frame, 20 dices of size $(14 \times 14 \times 14(\mathrm{~mm}))$ each. The robot arm has one degree of freedom, and is capable of carrying out pick and drop tasks, with the dices. In addition the robot arm may also serve as a counterbalance for the whole structure of the hovercraft. Using the robotic arm is most effective in situations where the aircraft demands autonomous task handling and weight modification during flight. Control perspective, this requires careful consideration by the governing party. The robot arm position is shown below the vehicle.

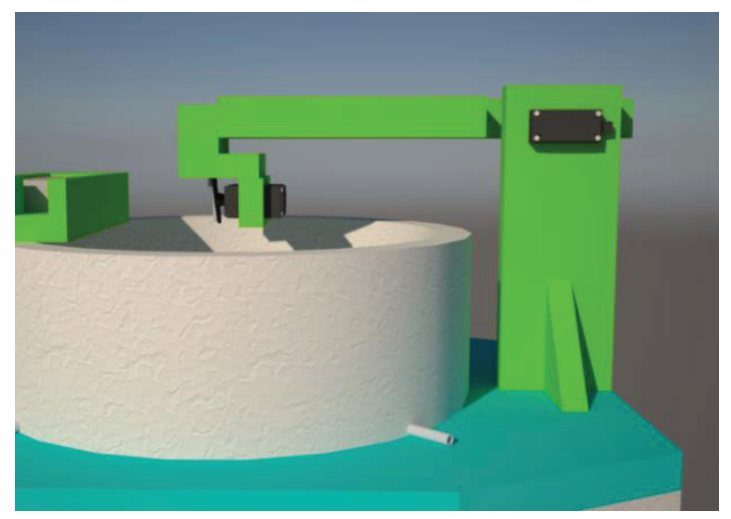

Fig. 2. The robot arm on the hovercraft

In terms of the physical parameters of the air-cushion vehicle has the following: a hovercraft including cube size: $480(\mathrm{~mm})$ long, $380(\mathrm{~mm})$ wide, $330(\mathrm{~mm})$ high (with robot arm state shown in fig. 2.). The mass is small relative to its dimensions. With the battery and dices, the total weight of vehicle is $1.27(\mathrm{~kg})$.

The two hoist motors and the forward force providing one are the same type, A2212 / 13T brushless DC motors. They have the following parameters [4] :

Motor weight: 52.7 (grams), the maximum operating voltage: 11.1 (V) (Lithium-Polymer battery cell 3) maximum operating current: 10 (A), the maximum achievable operating power (non-continuous operation): 150 (W), shaft diameter: 3.2 (mm), speed Kv: 1000 (RPM / V). The brushless DC motors with high efficiency (over $80 \%$ ) and a high power / weight ratio. The robot arm with two MG995type servo motor is housed.

One of the joints of the robotic arm provides the robotic arm movement, and the other will ensure the functioning of the mechanism performing the removal of the dice. The servos as data having the following main characteristics [5]:

Weight: 55 (grams), the maximum operating voltage $7.2(\mathrm{~V})$, the maximum torque of $0.98(\mathrm{Nm})$ capable of rotating 180 (degrees), can be positioned within this range.

\section{The circuit of hovercraft}

The circuit was designed in $\mathrm{KiCAD}$ [6], the main part is the PIC18F type microcontroller. This controls the brushless motors with production of the PWM (Pulse Width Modulation) signal, controls the robot arm servo motors, measures the battery voltage level and communicates via Bluetooth module to the control computer.

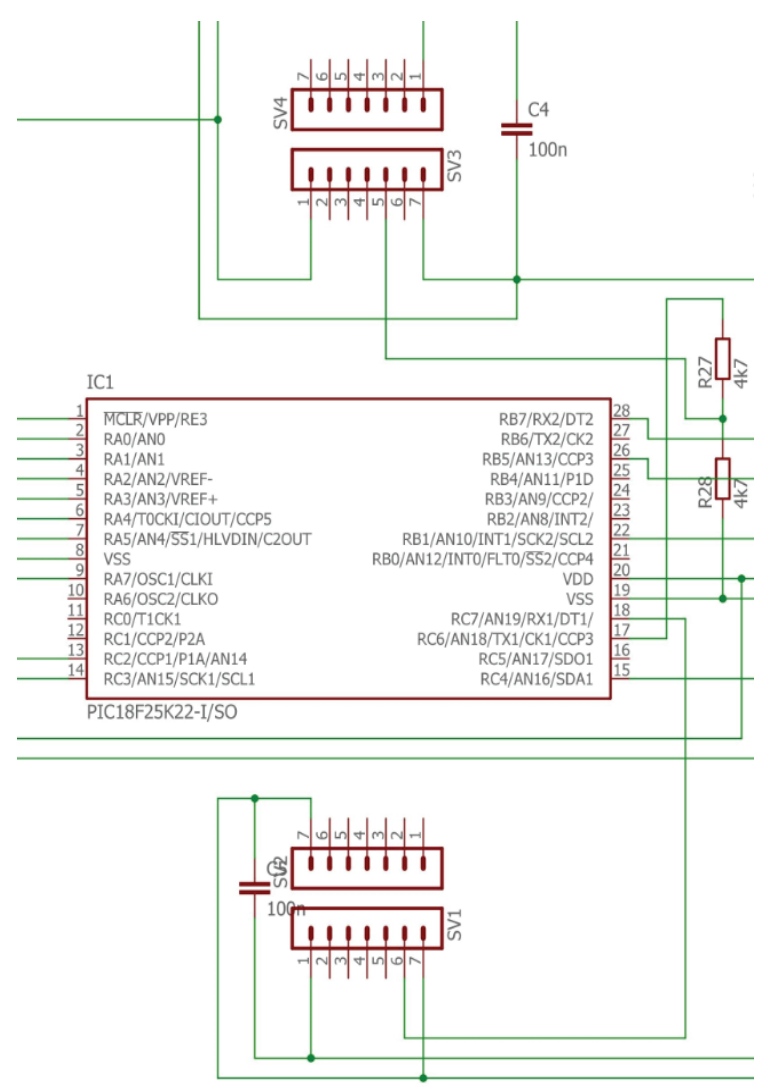

Fig. 3. Connection of the microcontroller used for Hovercraft

The Bluetooth module type was HC-05. This will allow two-way communication, so can be transmitted to the control computer the battery voltage and the position of servo motors of the robot arm. The battery type used capable of 3-cell Li-Po (Lithium-polymer) battery of 2200 (mAh) and 11.1 (V) voltage delivery. It fell Li-Po battery of choice, as the ratio of capacity / weight applied to large, making it ideal for remote-controlled vehicles.

Furthermore, large currents are able to deliver. The circuit is housed in two voltage stabilizer integrated circuits. One provides $3.3(\mathrm{~V})$ voltage supply necessary

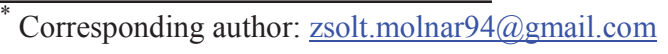


for the Bluetooth module and the other necessary stable $5(\mathrm{~V})$ power supply for the microcontroller.

The power required for the production of servo motors, DC brushless motors with control electronics provides, because they have a built-in 5 (V) voltage stabilizer and able to lead a 1 (A) current.

\section{The construction of hovercraft and material selection}

During the build-out should strive to get the materials used are lighter, but they should have a high resistance against arise in the performance of their functions and management of conflicts of mistakes. Based on these considerations, the choice fell polystyrene insulation versions of different densities. Indicatively three different density polystyrene have been used.

The completed 3D model allows you to weigh the volume of polystyrene various types of air-cushion vehicle designed to scale.

Thus, knowing the density of polystyrene [7], calculate the mass of the vehicle frame even before designing.

The following table provides a summary of the different types of polystyrene density and volume of this calculated mass used.

Table 1. The mass of vehicle

\begin{tabular}{|lccc|}
\hline & Density $\left(\mathrm{g} / \mathrm{cm}^{3}\right)$ & Volume $\left(\mathrm{cm}^{3}\right)$ & $\begin{array}{c}\text { Mass } \\
(\mathrm{g})\end{array}$ \\
\hline $\begin{array}{l}\text { Expanded } \\
\text { polystyrene (White) }\end{array}$ & 0.015 & 7490 & 112,35 \\
\hline $\begin{array}{l}\text { Extruded (Blue) } \\
\text { polystyrene (Blu) }\end{array}$ & 0.03 & 3073 & 92.19 \\
\hline $\begin{array}{l}\text { Expanded (Green) } \\
\text { polystyrene (Gren }\end{array}$ & 0.052 & 815 & 42.38 \\
\hline
\end{tabular}

The summing calculated values in the table, the main body of the air mass of $246.92(\mathrm{~g})$, which is added to the electronics, motors, fasteners, adhesives, dice weight, battery used. The total weight of the assembled hovercraft is $1.27(\mathrm{~kg})$. The polystyrene has high physical resistance, low density and can be well machined.

The foam cutter uses a glowing thin electrical conductor, which may be of polystyrene cut to size even and smooth cuts. As our own design with the help of CNC machine, which also functions as a foam cutter. The 3D plans have been required to file generating CNC control, then executed the finished polystyrene parts.

Such assembly has polystyrene glue [8] and by means of hot melt [9] is performed.

\section{Remote control and software}

The remote control of the hovercraft Bluetooth HC-05 module has been used, which can directly communicate with Bluetooth module built into laptops or commercially available Bluetooth adapter with a USB (Universal Serial Bus) connection. In the project a Dell Inspiron Mini 1018 netbook has been carrying out the tests and was used for control because of the mobility and long battery life.

The netbook has the following specifications [10]:

1) Processor: Intel Atom N455 $1.66(\mathrm{GHz})$

2) $R A M: 1(G B) D D R 3$

3) Display: 10 ", $1366 \times 768$ resolution

4) Video card: Integrated Intel NM10 Express

5) Storage: $250(\mathrm{~GB}) \mathrm{HDD}, 5400$ (RPM)

6) Weight: $1.33(\mathrm{~kg})$

7) Battery capacity: 48 (Wh)

8) Built-in Bluetooth 2.1 and 802.11b / g WiFi (Wireless Fidelity)

These specifications make it suitable to run a hovercraft made software to control and communicate with it via Bluetooth. The software has been designed so that users can directly from the computer control the vehicle or can take control over by using a glove with accelerometer.

The glove is measuring the hand tilt and sends out this data to the computer software which generates the control data for the vehicle microcontroller.

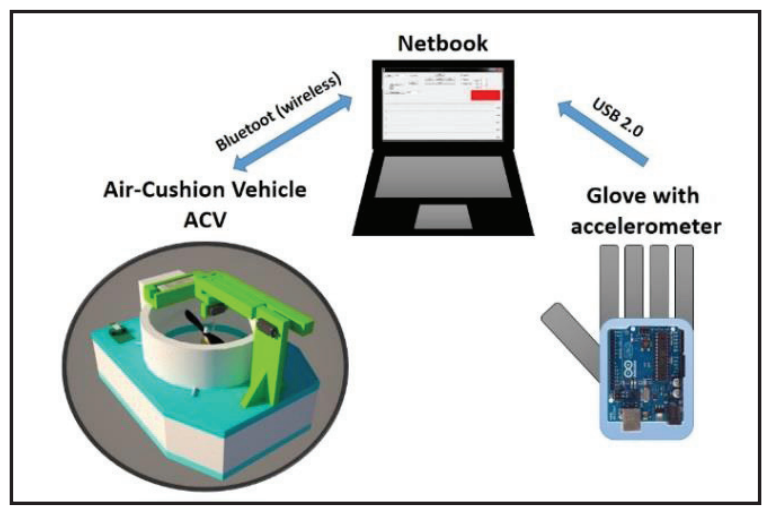

Fig.4. Remote control concept

The figure shows that the netbook communicates wirelessly via Bluetooth. The acceleration sensor glove is directly connected to the notebook via a USB cable. The acceleration sensor type used is MMA7361 sensor three-axis, micro-machined type. The sensitivity can be adjusted $\pm 1.5(\mathrm{~g})$ and $\pm 6(\mathrm{~g})$. The $(\mathrm{g})$ value means that many times the acceleration of gravity will be the maximum measured acceleration value. The gravitational acceleration is $9.81(\mathrm{~m} / \mathrm{s} 2)$.

For tilt measurement we consider that it points to the Earth's surface perpendicular to the sensor direction and heeling 1 (g) acceleration, which is the Earth's gravitational field distributed to varying degrees among the three axes. From these values can be determined the orientation of the glove thus the orientation of the operator hand with respect to the Earth's surface. For the control of the air-cushion vehicle was used $1.5(\mathrm{~g})$ of sensitivity range to achieve the high sensitivity of the sensor. The output of analog signals, varies from 0 (V) to $3.3(\mathrm{~V})$ range. These signals must be converted to digital values prior processing.

For the sake of completion of an analog to digital conversion has been Arduino UNO [11] used. It has the

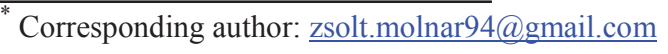


required analog inputs and a USB connection in order to connect to the netbook.

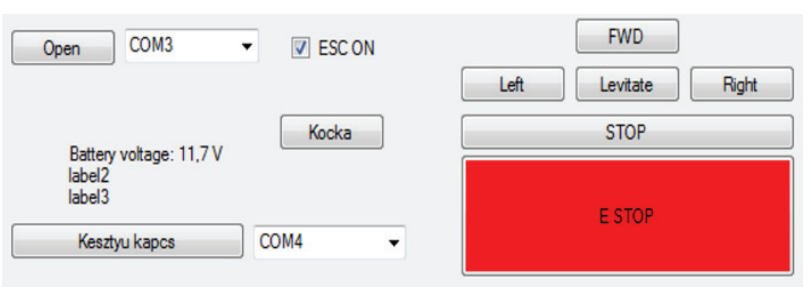

Fig.5. The GUI (Graphical User Interface) of the controller

The main task of the software running on the computer is to provide continuous communication between the computer and the hovercraft. It is important that in case of loss of communication, the hovercraft controller will detect and control the motors automatically to shut down for safety. Since the hovercraft and acceleration sensing glove, both are treated as serial port by the computer, so we have two serial communication port selection drop-down list on the GUI. These are 'COM3' and 'COM4' by default.

The interface also reads the actual battery voltage, which is important in case of Li-Po batteries since the excessive discharge leads to damage. The button and a stop button enabling control in four directions housed in the user interface, if you want to control your vehicle from your computer.

In addition, it is equipped with an 'E-STOP' (Emergency-STOP) button on the user interface which performs an immediate emergency stop, the motors shuts down and interrupts the communication.

The adjustable 'Levitate', 'Turn', 'FWD' are the values of the maximum power of the motors, which operate while floating or turning. The turning is higher value, because in such a case, only one lift motor functions due to the use of the torque to turn and it must ensure the inoperative motor performance as well. In addition, the program has three control modes. The first is the 'cushion'. In this mode, with the tilt of the glove the motors can be switched on and off in order to rotate the hovercraft. There are no transitional values, the inclination of the glove is only used by the software for thresholds that the engine is turned on or off.

This control mode is recommended when walking next to the hovercraft and cannot be guaranteed the stability of the governing hand of the person. The following is the 'Robot arm', in this case with the tilt of the glove can be varied the inclination of the robot arm. The third mode is the 'Analog Control' when it comes to managing people can keep their hands firmly and properly in this mode, the software controls the engine speed to the degree of inclination of the glove.

The control software is written for Windows 7 64-bit operating system, the software is in $\mathrm{C}$ \# using Visual Studio [12] development environment that is free for student use.

\section{Conclusion}

The planned hovercraft was built and tested. Functionally, as expected, it is difficult to control, the ability to move with great speed, it requires attention, as in the case of quadrotors.

During testing, smaller collisions occurred, which causes no damage in vehicle frame due to the polystyrene impact absorbing ability.

The remote control works in terms of a stable, open space of $30 \mathrm{~m}$ range is also available with it.

In terms of battery life depending on terrain, indoors, where the floor is smooth to operate the hoist motors small amount of power is required, but outdoors more power is needed in order to avoid any uneven ground.

In summary it can be said that it meets the expected conditions, however, with further software development various flight situations can be implemented, such as the simulation of air turbulence for quadrotors becomes possible.

The project was supported by University of Debrecen, Department of Electrical Engineering and Mechatronics.

\section{References}

[1] G. Husi, "Industry 4.0 technology in University of Debrecen", https://www.researchgate.net/publication/301607839_Industry_4 0 _Hungarian, accessed on May 2017

[2] S.A.M. Ali, "Guidance, Navigation, modeling and control of a hovercraft", https://www.researchgate.net/publication/ 277776432_Guidance_Navigation_modeling_and_control_of_a_ hovercraft, accessed on February 2017

[3] SketchUp Pro, http://www.sketchup.com download?sketchup=pro, accessed on January 2017

[4] A2212/13T TECHNICAL DATA, http://www.rhydolabz.com/ documents/26/BLDC_A2212_13T.pdf, accessed on April 2017

[5] MG995 High Speed Metal Gear Dual Ball Bearing Servo, http://www.electronicoscaldas.com/datasheet/MG995_TowerPro.pdf, accessed on April 2017

[6] KiCad EDA, http://kicad-pcb.org/, accessed on January 2017

[7] Kunststoffe international, Rigid Polystyrene Foam (EPS, XPS), http://www.plasticsportal.net/wa/plasticsEU it IT/function/conv ersions:/publish/common/upload/technical_journals/plastics_tren dreports/Rigid_polystyrene_foam.pdf, accessed on February 2017

[8] Polystyrene Foam Adhesive, http://www.genkem.co.za/genkem/ index.php/diy-with-genkem/item/polystyrene-foam-adhesive, accessed on March 2017

[9] Hot Melt Glue Gun, https://www.amazon.com/CCbetterTemperature-Melting-Flexible-Projects/dp/B01178RVI2, accessed on February 2017

[10] Dell Inspiron Mini 1018, http://www.dell.com/enus/shop/productdetails/inspiron-mini1018, accessed on April 2017

[11] Arduino UNO \& Genuino UNO https://www.arduino.cc/en/main/arduinoBoardUno, accessed on April 2017

[12] Microsoft, Visual Studio IDE, https://www.visualstudio.com/, accessed on March 2017 